\title{
Commentary: Chemoradiotherapy for locally advanced squamous cell carcinoma of the oropharynx: Does completion of systemic therapy affect outcomes?
}

\author{
Richard Sleightholm' and Michael J Baine ${ }^{2 *}$ \\ 'Department of Pharmaceutical Sciences, University of Nebraska Medical Center, Omaha, NE, USA \\ 2*Department of Radiation Oncology, Fred and Pamela Buffett Cancer Center, University of Nebraska Medical Center, Omaha, NE, USA
}

Article Info

Article Notes

Received: February 07, 2018

Accepted: March 23, 2018

\section{*Correspondence:}

Dr. Michael J. Baine, MD, PhD

Department of Radiation Oncology

Fred \& Pamela Buffett Cancer Center

University of Nebraska Medical Center

987521 Nebraska Medical Center

Omaha, NE 68198-7521, USA;

Telephone: 402-552-3844 (office), Fax: 402-552-7799,

E-mail: mbaine@unmc.edu

(c) 2018 Baine MJ. This article is distributed under the terms of the Creative Commons Attribution 4.0 International License.

\section{ABSTRACT}

Concurrent chemoradiotherapy plays a vital role in the treatment of locally advanced squamous cell carcinoma of the oropharynx (LA-OPC). Importantly, only $50-85 \%$ of patients undergoing this treatment are able to complete their prescribed chemotherapy courses due to treatment-associated morbidity. The significance of chemotherapy completion, or lack thereof, remains unknown in this setting. To further investigate if chemotherapy non-completion affects patient outcomes, a single institutional retrospective study was undertaken analyzing 73 patients undergoing definitive radiation for LA-OPC and revealed a near-significant trend toward improved overall survival on multivariable analysis $(p=0.053)$ in those completing chemotherapy. We are presently providing a commentary on this manuscript in an attempt to provide context and further interpretation of the presented results as well as suggest areas in need of additional investigation.

Treatment options for locally advanced squamous cell carcinoma of the oropharynx (LA-OPC) consistently includes radiation therapy either in an adjuvant or definitive setting ${ }^{1-2}$. When used definitively, clinical trials have demonstrated that the addition of concurrent systemic therapy confers a significant advantage in patient outcomes; improving local control, disease-free survival, and overall survival ${ }^{3-9}$. This concurrent therapy often consists of high-dose cisplatin $\left(100 \mathrm{mg} / \mathrm{m}^{2}\right)$ delivered every 3 weeks for a total of 3 doses provided over the course of the radiation treatment, but low dose cisplatin consisting of weekly doses of $30-40 \mathrm{mg} / \mathrm{m}^{2}$ or daily doses of $6 \mathrm{mg} / \mathrm{m}^{2}$, carboplatin, or cetuximab can be substituted in patients who will not tolerate high-dose cisplatin ${ }^{10-13}$.

Importantly, utilization of systemic therapy concurrent with definitive radiation treatment results in a significant increase in acute treatment-associated morbidity, leading to increased marrow suppression, nausea, fatigue, and mucositis ${ }^{14-16}$. The treatmentassociated mucositis, particularly, often causes considerable pain leading patients to require high-dose narcotics as well as suffer from poor oral intake and consequent malnutrition. Unfortunately, for between $15-50 \%$ of patients, these acute toxicities prevent completion of their chemotherapy regimens ${ }^{17-20}$.

The lack of chemotherapy completion in the setting of 
definitive concurrent chemoradiotherapy for LA-OPC remains of unknown significance. Generally, based on the results of RTOG 0129 , most physicians believe that patients completing at least 2 of the planned 3 cycles of high-dose cisplatin is sufficient. This trial, comparing hypofractionated versus conventionally fractionated radiation regimens concurrent with 2 or 3 cycles of highdose cisplatin, respectively showed no significant difference in outcomes between these treatment groups nor was there a significant difference in outcomes in patients receiving 2 versus 3 cycles of concurrent cisplatin in the conventionally fractionated arm (though those receiving 1 cycle did significantly worse) ${ }^{21-22}$. Importantly, however, the patient cohort receiving only 2 of their planned 3 cycles of cisplatin contained only 86 patients, and though not statistically significant, those who received all 3 cycles of chemotherapy had a trend towards improved overall survival when compared to those completing only 2 cycles. Additionally, recent meta-analysis has indicated that overall survival is directly associated with increased cumulative dose of cisplatin received in non-nasopharyngeal squamous cell carcinoma of the head and neck ${ }^{23}$. With the lack of a clear association between chemotherapy completion and treatment outcomes in LA-OPC patients undergoing definitive concurrent chemoradiotherapy, our group attempted to shed more light on this topic through a retrospective study ${ }^{24}$.

In total, we retrospectively analyzed the records of 73 patients undergoing definitive radiation therapy with concurrent systemic agents at out institution from 20072014 . In our population, most patients were male $(87.7 \%)$, had an ECOG performance status of 0-1 (90.4\%), were HPV positive/HPV unknown (63.0\%/26.0\%), and had locally advanced disease due to nodal involvement as opposed to local tumor invasion (54.8\%). Most patients (97.3\%) completed all prescribed radiation therapy. Systemic therapy was not limited to high-dose cisplatin only but rather additionally included patients who received weekly cetuximab (20.5\% of the included patients) and weekly cisplatin $(2.7 \%$ of the included patients). At a median follow-up of 3.4 years, completion of systemic therapy did not seem to affect rates of locoregional failure $(p=0.93)$ though did show a trend toward improving distant failure rates by $13.0 \%$ (20.0\% vs $7.0 \%, p=0.12)$ and overall survival by $18.8 \%$ ( $82.1 \%$ vs $63.3 \%, p=0.08$ ). Due to incomplete records, we were not able to calculate cumulative doses and perform a stratified analysis similar to the previous work of Strojan et al. ${ }^{23}$ On multivariate analysis, the trend toward improved overall survival with systemic therapy completion was more apparent still (Hazard Ratio $=0.388$; $\mathrm{p}=0.053)^{24}$.

Accounting for the low patient numbers included in our analysis and consistency with results from RTOG 0129 , the near-significant trend toward improved outcomes, particularly improved overall survival, with systemic therapy completion is interesting. Further, the remarkable reduction in hazard for death (0.39) with systemic therapy completion revealed in our study increases the likelihood that this trend, if true, may be of high clinical significance. Further, though past data has only hinted at this trend of improved outcomes being present in the context of concurrent high-dose cisplatin, our study suggests that it may indeed be true across all concurrent systemic therapy regimens in patients treated with definitive radiation therapy for LA-OPC.

We postulate that the near-significant trend toward improved survival in patients who complete systemic therapy may be due to the observed trend toward increased distant relapse in non-completers. This suggests that completion of systemic therapy may be more important for eradicating microscopic distant metastatic disease as opposed to improving radiosensitization, particularly as systemic therapy completion had no effect on locoregional control. Alternatively, however, it is possible that the observed effect of systemic therapy non-completion decreasing overall survival is confounded by patient comorbidities resulting in both poor tolerance to systemic therapy and increased risk of death. Unfortunately, due to study design, we were unable to fully account for this possibility.

With the obvious pitfalls associated with the retrospective design and limited patient number included in our study, we believe that our results warrant further study using either a larger, multi-institutional retrospective database or through further subgroup analyses of prospective clinical trials. If undertaken in a de novo prospective setting, we would suggest inclusion of not only total cumulative doses of systemic agents administered but also various pharmacokinetic parameters such as blood levels and elimination kinetics which may better elucidate how much, how often, and how long we should administer the respective systemic agents to achieve an optimal balance of improved tumor response and reduced toxicity. Furthermore, parameters of immunotherapy or other established treatments administered should similarly be investigated so that we have a fuller understanding of both the complete drug regimens and individual therapies. For example, $5 / 15$ patients receiving cetuximab in our study discontinued use because of side effects. However, the nature and severity of these complications remain unclear due to incomplete records. Thus a prospective study describing the rate and degree of severity of adverse events would be of great utility in guiding clinical decision making. Also of importance, any further study of this nature must also be contextualized on the pre-existing comorbidities, specific cause of systemic therapy cessation, and cause 
of death for the included patients to allow confounders to be better accounted for and thus determine if any associated trend is causative or only correlative. Further, as our study is the first to our knowledge to investigate the effects of systemic therapy completion including non-highdose cisplatin regimens in oropharyngeal cancers, others have published similar studies investigating cumulative chemotherapy dose delivered and various alternative chemotherapy regimens in head and neck cancers ${ }^{13,23 \text {, }}$ 25. As such, a more robust investigation is warranted regarding systemic therapy completion in LA-OPC patients undergoing alternative systemic therapy regimens including weekly cisplatin or cetuximab.

\section{References}

1. Head and Neck Cancers, Version I.2016. NCCN Clinical Practice Guidelines in Oncology. www.nccn.org. Accessed July 12, 2016.

2. Ang KK, Garden AS. Radiotherapy for Head and Neck Cancers. Indications and Techniques, Fourth Edition. Lippincott Williams \& Wilkins, 2012.

3. Pignon JP, Bourhis J, Domenge C, et al. Chemotherapy added to locoregional treatment for head and neck squamous cell carcinoma: Three meta-analyses of updated individual data. Lancet. 2000; 355:949-955.

4. Wendt TG, Grabenbauer CG, Rodel CM, et al. Simultaneous radiochemotherapy versus radiotherapy alone in advanced head and neck cancer: A randomized multicenter study. J Clin Oncol. 1998; $16: 1318-1324$

5. Calais G, Alfonsi M, Bardet E, et al. Randomized study comparing radiation alone RT versus concomitant chemotherapy and radiation therapy for advanced-stage oropharynx carcinoma. J Natl Cancer Inst. 1999; 91:2081-2086

6. Adelstein DA, Lavertu P, Saxton JP, et al. Mature results of a phase III trial comparing concurrent chemoradiotherapy with radiation therapy alone in patients with stage III and IV squamous cell carcinoma of the head and neck. Cancer. 2000; 88:876-883.

7. Olmi P, Crispino S, Fallai C, et al. Locoregionally advanced carcinoma of the oropharynx: Conventional radiotherapy vs. accelerated hyperfractionated radiotherapy vs. concomitant radiotherapy and chemotherapy - a multicenter randomized trial. Int J Radiat Oncol Biol. 2003; 55:78-92.

8. Budach V, Stuschke M, Budach W, et al. Hyperfractionated accelerated chemoradiation with concurrent fluorouracil-mitomycin is more effective than dose-escalated hyperfractionated accelerated radiation therapy alone in locally advanced head and neck cancer: Final results of the radiotherapy cooperative clinical trials group of the German Cancer Society 95-06 prospective randomized trial. J Clin Oncol. 2005; 23:1125-1135.

9. Bensadoun RJ, Benezery K, Dassonville 0, et al. French multicenter phase III randomized study testing concurrent twice-a-day radiotherapy and cisplatin/5-fluorouracil chemotherapy (BiRCF) in unresectable pharyngeal carcinoma: Results at 2 years (FNCLCCGORTEC). Int J Radiat Oncol Biol Phys. 2006; 64:983-994.

10. Forastiere AA, Zhang $Q$ Weber RS, et al. Long-term results of RTOG 91-11L a comparison of three nonsurgical treatment strategies to preserve the larynx in patients with locally advanced larynx cancer. J Clin Oncol. 2013; 31(7):845-52.

11. Chan AT, Leung SF, Ngan RK, et al: Overall survival after concurrent cisplatin-radiotherapy compared with radiotherapy alone in locoregionally advanced nasopharyngeal carcinoma. J Natl Cancer Inst. 2005; 97(7):536-9.

12. Jeremic B, Shibamoto Y, Stanisavljevic B, et al: Radiation therapy alone or with concurrent low-dose daily either cisplatin or carboplatin in locally advanced unresectable squamous cell carcinoma of the head and neck: A prospective randomized trial. Radiother Oncol. 1997; 43(1):29-37.

13. Bonner JA, Harari PM, Giralt J, et al. Radiotherapy plus cetuximab for squamous cell carcinoma of the head and neck. NEJM. 2006; 354:567-78

14. Vera-Llonch M, Oster G, Hagiwar M, et al. Oral mucositis in patiests undergoing radiation treatment for head and neck carcinoma: risk factors and clinical consequences. Cancer 2006; 106(2):329-36.

15. Machtay M, Moughan J, Trotti A, et al. Factors associated with severe late toxicity after concurrent chemoradiation for locally advanced head and neck cancer: an RTOG analysis. J Clin Oncol. 2008; 26(21):3582-9.

16. Adelstein DJ. Oropharyngeal cancer: the role of chemotherapy. Curr Treat Options Oncol. 2003; 4(1):3-13.

17. Fayette J, Molin Y, Lavergne E, et al. Radiotherapy potentiation with weekly cisplatin compared to standard every 3 week cisplatin chemotherapy for locoregionally advanced head and neck squamous cell carcinoma. Drug Design, Development, and Delivery. 2015; 9:6203-10.

18. Bahig H, Fortin B, Alizadeh M, et al. Predictive factors of survival iand treatment tolerance in older patients treated with chemotherapy and radiotherapy for locally advanced head and neck cancer. Oral Oncology. 2015; 51:521-28.

19. Krstevska V, Stojkovski I, Zafirova-Ivanovska B. Concurrent radiochemotherapy in locally-regionally advanced oropharyngeal squamous cell carcinoma: analysis of treatment results and prognostic factors. RO. 2012; 7:78

20. Adelstein DJ, Adams GL, Wagner $\mathrm{H}$, et al. An Intergroup phase III comparison of standard radiation therapy and two schedules of concurrent chemoradiotherapy in patients with unresectable squamous cell head and neck cancer. J Clin Oncol. 2003; 21:92-98.

21. Ang KK, Harris J, Wheeler R, et al. Human papillomavirus and survival of patients with oropharyngeal cancer. NEJM. 2010; 363:24-35.

22. Nguyen-Tan PF, Zhang Q And KK, el al. Randomized phase III trial to test accelerated versus standard fractionation in combination with concurrent cisplatin for head and neck carcinomas in the Radiation Therapy Oncology Group 0129 trial: long-term report of efficacy and toxicity. J Clin Oncol. 2014; 32:3858-67.

23. Strojan P, Vermorken JB, Beitler JJ, et al. Cumulative cisplatin dose in concurrent chemoradiotherapy for head and neck cancer: A systematic review. Head \& Neck. 2016; 38:E2151-58.

24. Baine MJ, Dorius T, Bennion N, et al. Chemoradiotherapy for locally advanced squamous cell carcinoma of the oropharynx: Does completion of systemic therapy affect outcomes. Oral Oncology. 2017; 73:105-110.

25. Harari PM, Harris J, Kies MS, et al. Postoperative chemoradiotherapy and cetuximab for high-risk squamous cell carcinoma of the head and neck: Radiation Therapy Oncology Group RTOG-0234. J Clin Oncol. 2014; 32(23) 2486-95. 